\title{
Finite-Size Effects for Anisotropic Bootstrap Percolation: Logarithmic Corrections
}

\author{
Aernout C.D. van Enter · Tim Hulshof
}

Received: 6 February 2007 / Accepted: 2 July 2007 / Published online: 7 August 2007

(C) Springer Science+Business Media, LLC 2007

\begin{abstract}
In this note we analyse an anisotropic, two-dimensional bootstrap percolation model introduced by Gravner and Griffeath. We present upper and lower bounds on the finite-size effects. We discuss the similarities with the semi-oriented model introduced by Duarte.
\end{abstract}

Keywords Bootstrap percolation · Cellular automaton · Finite size effects · Metastability

\section{Introduction}

Bootstrap percolation (BP) models, sometimes also called k-core percolation or threshold growth models, are simple Cellular Automata with a percolation configuration chosen as a random initial condition. The deterministic dynamics is given by repeatedly applying a growth rule, and the question of interest is if the whole system will be occupied in the long run. They have been a topic of interest in fields as diverse as physics-for the study of magnetic models, metastability, rigidity, fluid flow in porous media and glassy dynamicsmathematics, computer science, neural science and economics, see e.g. $[1,4-12,16-18$, 20-23, 26-28, 30].

Most of the models which have been considered in the literature are isotropic. In this paper we consider an anisotropic model in two dimensions which was introduced by Gravner and Griffeath $[14,15]$. Their model is critical, meaning that there are infinite sets with an infinite complement which can fill the entire lattice, but no finite set can do so, and all sets with a finite complement will fill up the lattice. It has a trivial percolation threshold $p_{c}=0$. We determine the asymptotic behaviour of the finite-size effects, and find it to be

\footnotetext{
A.C.D. van Enter ( $\varangle)$

Institute for Mathematics and Computing Sciences, Rijksuniversiteit Groningen, Blauwborgje 3, P.O. Box 800, 9700 AV Groningen, The Netherlands e-mail: aenter@phys.rug.nl

T. Hulshof

Star Numanstraat 93 b, 9714 JM Groningen, The Netherlands

e-mail: W.J.T.Hulshof@student.rug.nl
} 
different from the one proposed by Gravner and Griffeath. We discuss the similarity with the behaviour of the semi-oriented (Duarte) model which is also anisotropic, and the behaviour of which was established by Mountford [2, 3, 13, 24, 25, 29].

\section{Definition and Some Known Properties of Bootstrap Percolation Models}

We will consider BP models either on $\mathbb{Z}^{2}$ or on finite subsets thereof.

The dynamics is defined as follows:

- Once a site is occupied, it will remain so forever.

For each empty site it is checked at each time step whether in a prescribed neighborhood $x+\mathcal{N}$ of the site $x$ at least $\theta$ sites are occupied. (The parameter $\theta$ is called the threshold value.) If so, at the next time step the site will be occupied.

- At the next time step this procedure is repeated.

Thus this updating rule provides a deterministic, parallel dynamics. This dynamics runs until no changes occur anywhere anymore.

To describe the evolution of an occupied set $A \subset \mathbb{Z}^{2}$ we define the discrete time step operator $\mathcal{T}$ :

$$
\mathcal{T}(A)=A \cup\{x:|(x+\mathcal{N}) \cap A| \geq \theta\} .
$$

Starting from some initial set $A_{0} \subset \mathbb{Z}^{2}$, we iterate: $\mathcal{T}\left(A_{n}\right)=A_{n+1}$. We also define $A_{\infty}=$ $\mathcal{T}^{\infty}\left(A_{0}\right)=\bigcup_{n=0}^{\infty} A_{n}$. We call a model critical if there exists some infinite $A_{0}$ (but no finite one) with an infinite complement in $\mathbb{Z}^{2}$ such that $A_{\infty}=\mathbb{Z}^{2}$, that is the dynamics eventually fills all of $\mathbb{Z}^{2}$.

We choose for our random initial configuration a percolation configuration, where each site is independently occupied with probability $p$.

The standard (isotropic) model is defined by taking as the neighborhood of a site its four nearest neighbours and $\theta=2$.

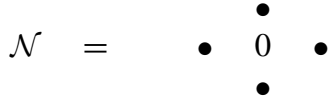

In that case $p_{c}=0$ for the infinite lattice [31] (so for each initial density the lattice will fill up). The finite-size effects at small $p$ are such that if the size of a square is of order $\exp \left(C_{1} \times \frac{1}{p}\right)$, with $C_{1} \geqslant C s t$ for an explicit, computable constant $C s t$, the square tends to be occupied in the end, while if it is of order $\exp \left(C_{2} \times \frac{1}{p}\right)$, with $C_{2} \lesseqgtr C s t$, it tends to remain mostly empty $[5,17]$ with overwhelming probability.

In the Gravner-Griffeath (GG) model, the neighborhood of a site consists of six sites, to wit, the closest two sites to the East, the closest two sites to the West, and the nearest neighbours in the North and South direction. The threshold value $\theta$ now is defined to be 3 .

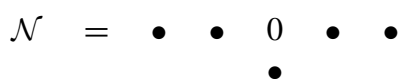

For later reference we also mention the Duarte (semi-oriented) BP model, in which the neighborhood of a site consists of three sites, namely its North, West and South neighbours, and $\theta$ is chosen to be 2 .

$$
\mathcal{N}=\bullet 0
$$


This model can not grow in the Western direction.

The GG model (similarly to the Duarte model) has easy and hard growth directions. Indeed, once an $N$ by $N$ square is occupied, a single occupied site at distance 1 or 2 will fill the next line segment, in the East or West direction, while for growth in the North or South direction two sites with no more than 3 empty sites in between need to be occupied, which is much less probable (locally the probability is of order $p^{2}$ instead of order $p$ ).

Two important notions in the theory are those of a "critical droplet" (sometimes called nucleation droplet) and a volume being "internally spanned".

A critical droplet is an occupied configuration which will keep on growing with large probability. In the regular model, for example, an occupied square with side length $C_{1} \times \frac{1}{p}$, $C_{1}$ large, has this property, while in the Duarte model, an occupied line segment of length $C_{1} \times \frac{1}{p} \times \ln \frac{1}{p}, C_{1}$ large, is known to have this property.

The second important notion is that of a volume being internally spanned. We will call a volume $V$ 'internally spanned' if given some occupied set $A_{0} \subset V, A_{\infty}$ occupies all the sites in $V$. In that case the initially occupied set $A_{0}$-which typically will be a random setspans the volume $V$. (In the Duarte model the notion of internally spanned is defined slightly differently.)

Remark A related quantity, for which a similar scaling behaviour can be derived, as a corollary of our results, is the first passage time at the origin, that is the time at which the origin is occupied for the first time, see e.g. [5, 14].

\section{Main Result and Proof}

Our main result is that the volume size for which the Gravner-Griffeath model changes from typically not being internally spanned to typically being internally spanned scales as $\exp \left(O\left(\frac{1}{p} \times \ln ^{2}\left(\frac{1}{p}\right)\right)\right)$.

This contradicts a statement by these authors in $[14,15]$. Indeed we have:

\section{Theorem 1}

Consider the Gravner-Griffeath model.

(A) If a square is larger than $\exp \left(C_{1}\left(\frac{1}{p} \times \ln ^{2}\left(\frac{1}{p}\right)\right)\right)$, with $C_{1}$ large enough, it is internally spanned with large probability, for $p$ small enough.

(B) If a square is smaller than $\exp \left(C_{2}\left(\frac{1}{p} \times \ln ^{2}\left(\frac{1}{p}\right)\right)\right)$, with $C_{2}$ small enough, it is not internally spanned with large probability, for $p$ small enough.

Proof We will prove (A) and (B) separately.

To prove (A), we will first show that an occupied rectangle of size 2 by $C_{1} \times \frac{1}{p} \times \ln \frac{1}{p}$ is a critical droplet.

Indeed, let such a rectangle (a double segment, long in the North-South direction, width 2 in the East-West direction) be occupied, then we will first argue that with large probability it will grow in the Eastern and Western directions, until it fills up a rectangle of size $2\left(\frac{1}{p}\right)^{C}$ by $C_{1} \times \frac{1}{p} \times \ln \frac{1}{p}$ as long as $C$ is smaller than $C_{1}$. (When $C$ is larger than 1 , such a rectangle is much larger in the East-West than the North-South direction.)

For this to happen, it is sufficient that in all the $\left(\frac{1}{p}\right)^{C}$ line segments on the left and on the right at least one site is occupied in the original configuration. But this happens with 
probability

$$
\left(1-(1-p)^{C_{1} \frac{1}{p} \ln \frac{1}{p}}\right)^{O\left(\frac{1}{p}\right)^{C}} \simeq\left(1-p^{C_{1}}\right)^{O\left(\frac{1}{p}\right)^{C}},
$$

which is close to one when $C$ is smaller than $C_{1}$.

Once the larger rectangle is occupied, with $C$ chosen to be large enough (it should be at least larger than 2), it will grow in all directions, with large probability. Indeed, if we take the rectangle to be $N_{1}$ by $N_{2}, N_{1}$ much larger than $\frac{1}{p^{2}}$ and $N_{2}$ much larger than $\frac{1}{p}$, then the probability that the rectangle does not stop growing in either horizontal or vertical direction is larger than

$$
\prod_{k=1}^{\infty}\left(1-\left(1-p^{2}\right)^{N_{2}+k}\right)\left(1-(1-p)^{N_{1}+k}\right) .
$$

Minus the logarithm of this probability is approximately

$$
\sum_{k=1}^{\infty}\left(1-p^{2}\right)^{N_{2}+k}+(1-p)^{N_{1}+k}
$$

which is small, showing that the corresponding probability is close to 1 .

As the probability for a particular rectangle to be a critical droplet (thus the density of critical droplets) is larger than $p^{2 C_{1} \frac{1}{p} \ln \frac{1}{p}}=\exp \left(-2 C_{1} \frac{1}{p} \ln ^{2}\left(\frac{1}{p}\right)\right)$, the minimal size necessary for a square to contain with large probability a critical droplet is bounded from above by the inverse of this density. This critical droplet then will grow to fill up the entire square. Indeed, wherever in the square the droplet is, for small $p$ there will always be enough space to grow sufficiently far in at least two perpendicular directions (remember that the size of the square is much larger than $\frac{1}{p^{2}}$ ) and once the occupied set is big enough, with large probability it will then fill up its complement in the square.

(To derive this a bit more precisely, one can divide the large square into smaller subsquares. The occurrence of a critical droplet inside one such a subsquare is sufficient for growth with high probability. However, these occurrences now are independent between the different subsquares. Choose for example a subsquare of size $O\left(p^{-3}\right)$, as in the reasoning of [5], Lemma 5. Then a minor adaptation of their arguments provides the desired result.)

This finishes the proof of part (A).

To prove part (B), we essentially follow the analysis of Gravner and Griffeath [14], and point out where it needs to be modified. (Gravner and Griffeath based this part of their analysis largely on the proof of the similar statement for ordinary bootstrap percolation as given by Aizenman and Lebowitz in [5].)

We will first quote their definition and main claim:

Definition We call a rectangle $R$ in $\mathbb{Z}^{2}$ potentially internally spanned (PIS) if it is either a single site in $\Pi(p)$ or if (i) for every (integer) horizontal $\ell$ through $R$ there exist two sites $x, y \in \Pi(p) \cap R$ such that $\|x-y\|_{\infty} \leq 4$ and they are both at $\ell^{\infty}$-distance at most 4 from $\ell$, and (ii) every vertical line $\ell$ through it has a site in $\Pi(p) \cap R$ at $\ell^{\infty}$-distance at most 2 from $\ell$.

Here $\Pi(p)$ denotes the set of initially occupied sites, when the occupation probability is $p$. The property PIS is obviously weaker than that of being internally spanned. Indeed, a line that fails one of these two conditions can never be crossed, thus acts to block any growth across it and hence prevents the rectangle from being internally spanned. 
Claim Let $L<M$ be positive integers. Assume that the origin is not eventually occupied if the dynamics are restricted to $[-L, L]^{2}$, but is eventually occupied if the dynamics are restricted to $[-M, M]^{2}$. For every integer $a \in[4, L / 4]$, there exists a PIS rectangle $R$ included in $[-M, M]^{2}$ whose longest side is between $a$ and $4 a$.

The proof to the claim is given in [14].

From the definition of PIS it is then derived, replacing multiples of $p$ by $p$ at appropriate places, that the following must hold:

$$
\begin{aligned}
P(k, l) & :=P(\text { a fixed } k \times l \text { rectangle is PIS }) \\
& \leq \min \left\{\left(1-\left(1-p^{2}\right)^{k}\right)^{l},\left(1-(1-p)^{l}\right)^{k}\right\} \\
& :=\min \{I, I I\} .
\end{aligned}
$$

Note that for a fixed $l, I$ is increasing in $k$, whereas $I I$ is decreasing in $k$. We will thus look for $k$-values where these terms are of the same order, and an $l$-value which makes them as small as we can.

Now we deviate from [14]. We choose $k=\frac{1}{p^{3 / 2}}$, and $l=C_{2} \frac{1}{p} \ln \frac{1}{p}$, then we have bounds for both terms, namely

$$
\begin{aligned}
I I & =\left(1-(1-p)^{\frac{C_{2}}{p} \ln \frac{1}{p}}\right)^{\frac{1}{p^{3 / 2}}} \\
& \simeq\left(1-p^{C_{2}}\right)^{\frac{1}{p^{3 / 2}}} \\
& \simeq \exp \left(-\frac{p^{C_{2}}}{p^{3 / 2}}\right) \\
& \leq \exp \left(-C_{2} \frac{1}{p} \ln ^{2} \frac{1}{p}\right)
\end{aligned}
$$

if $C_{2}$ is small enough, and moreover

$$
\begin{aligned}
I & =\left(1-\left(1-p^{2}\right)^{\frac{1}{p^{3 / 2}}}\right)^{C_{2} \frac{1}{p} \ln \frac{1}{p}} \\
& \simeq\left(1-e^{-\sqrt{p}}\right)^{C_{2} \frac{1}{p} \ln \frac{1}{p}} \\
& \simeq p^{\frac{C_{2}}{2 p} \ln \frac{1}{p}} \\
& =\exp \left(-\frac{C_{2}}{2 p} \ln ^{2} \frac{1}{p}\right)
\end{aligned}
$$

which shows that for this choice of $k$ and $l$ and $C_{2}$ small enough

$$
P(k, l) \leq \exp \left(-\frac{C_{2}}{2 p} \ln ^{2} \frac{1}{p}\right) .
$$

These inequalities are the main ingredient in deriving the asymptotics which contradicts the claim on the asymptotics of Gravner and Griffeath. In particular they lead to a contradiction with their version of part (A). Their version of part (B) was not optimal and based on the choice $k=\frac{1}{p^{2}}$, and $l=\frac{1}{2} \frac{1}{p} \ln \frac{1}{p}$. With our choice of the pair $k, l$ and estimates as given above, the rest of their derivation provides the correct asymptotics. 
The choice of a small number $C_{2}$ in $l=C_{2} \frac{1}{p} \ln \frac{1}{p}$ in part (B) is consistent with the observation in part (A), that a large value of $C_{2}$ with high probability will allow growth in the East-West direction, as an interval of that length will not be all empty. Similarly, in our choice for $k$ we need that it is less than $O\left(p^{-2}\right)$, to block growth in the North-South direction with high probability. Instead of the power $p^{-3 / 2}$, any power between 1 and 2 in fact will do.

We suspect that in the derivation of Gravner and Griffeath, which they don't give completely but describe as a "straightforward computation", the following went wrong: They seem to have used the approximation $(1-x)^{N}=\exp (-x N)$ not only for small values of $x$, but also for larger $x$-values. For example, in an expression like $I$, with the choice $x=\left(1-p^{2}\right)^{\frac{1}{p^{3 / 2}}}$, and $N=C_{2} \times \frac{1}{p} \times \ln \frac{1}{p}$, the approximation gives a wrong answer, which is of the form proposed in [14]. For some further discussion on this point, and another derivation of part (A) of the theorem, see [19].

Thus if the size of a square is less than the inverse of the probability derived above, it will not be PIS with large probability. This is the main ingredient to prove (B). For further details see again [14].

\section{Discussion and Conclusions}

We have found that the asymptotics of the finite-size effects is different from the one proposed in [14]. In fact, the behaviour of the GG model is quite similar to that of the Duarte model.

It has the same asymptotics, and whereas the Duarte model has a single line segment of length $C_{1} \times \frac{1}{p} \times \ln \frac{1}{p}$, with $C_{1}$ large enough, as its critical droplet (first observed by Schonmann and communicated to the authors of $[2,3])$, in the GG model a double segment of the same length plays the same role. Therefore the difference between the two models suggested in [14] (one model understandable via its critical droplets, the other one via its growth mechanism) is in our opinion spurious. This observation leads us to expect that in considerable generality critical anisotropic BP models with slow and fast directions will have the same asymptotics for their finite-size effects.

Open questions It would be interesting to see if it is possible to identify the exact asymptotic constant (in other words, can $C_{1}$ and $C_{2}$ be chosen to be identical, as happens in the isotropic model [17]?).

Also it seems a natural question to see what the behaviour of anisotropic models in higher dimensions might be.

However, at present we have no results pertaining to these questions.

Acknowledgements We thank Christof Külske for a critical reading of the manuscript, and Alexander Holroyd for some helpful comments on the first version of our paper.

\section{References}

1. Adler, J.: Bootstrap percolation. Physica A 171, 452-470 (1991)

2. Adler, J., Duarte, J.A.M.S., van Enter, A.C.D.: Finite-size effects for some bootstrap percolation models. J. Stat. Phys. 60, 323-332 (1990) 
3. Adler, J., Duarte, J.A.M.S., van Enter, A.C.D.: Finite-size effects for some bootstrap percolation models, addendum. J. Stat. Phys. 62, 505-506 (1991)

4. Adler, J., Lev, U.: Bootstrap percolation: visualizations and applications. Braz. J. Phys. 33, 641-644 (2003)

5. Aizenman, M., Lebowitz, J.L.: Metastability effects in bootstrap percolation. J. Phys. A: Math. Gen. 21, 3801-3813 (1988)

6. Balogh, J., Bollobas, B.: Sharp thresholds in bootstrap percolation. Physica A 326, 305-312 (2003)

7. Biroli, G., Fisher, D.S., Toninelli, C.: Jamming percolation and glass transitions in lattice models. Phys. Rev. Lett. 96, 035702 (2006)

8. Biroli, G., Fisher, D.S., Toninelli, C.: Cooperative behavior of kinetically constrained lattice gas models of glassy dynamics. J. Stat. Phys. 120, 167-238 (2005)

9. Cerf, R., Cirillo, E.M.N.: Finite size scaling in three-dimensional bootstrap percolation. Ann. Probab. 27, 1833-1850 (1999)

10. Connelly, R., Rybnikov, K., Volkov, S.: Percolation of the loss of tension in an infinite triangular lattice. J. Stat. Phys. 105, 143-171 (2001)

11. De Gregorio, P., Lawlor, A., Bradley, P., Dawson, K.A.: Cellular automata with rare events; Resolution of an outstanding problem in the bootstrap percolation model. In: Cellular Automata. Amsterdam Proceedings, Lecture Notes in Computer Science, vol. 3305, pp. 365-374. Springer, Berlin (2004)

12. De Gregorio, P., Lawlor, A., Bradley, P., Dawson, K.A.: Clarification of the bootstrap percolation paradox. Phys. Rev. Lett. 93, 025501 (2004)

13. Duarte, J.A.M.S.: Simulation of a cellular automaton with an oriented bootstrap rule. Physica A 157, 1075-1079 (1989)

14. Gravner, J., Griffeath, D.: First passage times for threshold growth dynamics on $\mathbb{Z}^{2}$. Ann. Probab. 24, 1752-1778 (1996) (see also Griffeath's webpage: http://psoup.math.wisc.edu/kitchen.html)

15. Gravner, J., Griffeath, D.: Scaling laws for a class of cellular automaton growth rules. In: Proceedings 1998 Erdös Center Workshop on Random Walks, pp. 167-186 (1999)

16. Gravner, J., Holroyd, A.: Slow convergence in bootstrap percolation. arXiv: 0705.1347 (2007) (see also Holroyd's webpage: http://www.math.ubc.ca/holroyd/)

17. Holroyd, A.: Sharp metastability threshold for two-dimensional bootstrap percolation. Probab. Theory Relat. Fields 125, 195-224 (2003)

18. Holroyd, A.: The metastability threshold for modified bootstrap percolation in $d$ dimensions. Electron. J. Probab. 11, 418-433 (2006)

19. Hulshof, W.J.T.: The similarities between an unbalanced and an oriented bootstrap percolation model. Groningen bachelor thesis (2007)

20. Kozma, R., Puljic, M., Balister, P., Bollobas, B., Freeman, W.J.: Phase transitions in the neuropercolation model of neural populations with mixed local and nonlocal interactions. Biol. Cybern. 92, 367-379 (2005)

21. Kirkpatrick, S., Wilcke, W.W., Garner, R.B., Huels, H.: Percolation in dense storage arrays. Physica A 314, 220-229 (2002)

22. Lee, I.H., Valentiniy, A.: Noisy contagion without mutation. Rev. Econ. Stud. 67, 47-56 (2000)

23. Lenormand, R.: Pattern growth and fluid displacement through porous media. Physica A 140, 114-123 (1986)

24. Mountford, T.S.: Critical lengths for semi-oriented bootstrap percolation. Stoch. Proc. Appl. 95, 185-205 (1995)

25. Mountford, T.S.: Comparison of semi-oriented bootstrap percolation models with modified bootstrap percolation. In: Boccara, N., Goles, E., Martinez, S. (eds.) Cellular Automata and Cooperative Systems. NATO ASI Proceedings, pp. 519-525. Kluwer Acadamic, Dordrecht (1993)

26. Ritort, F., Sollich, P.: Glassy dynamics of constrained models. Adv. Phys. 52, 219-342 (2003)

27. Sabhapandit, S., Dhar, D., Shukla, P.: Hysteresis in the random-field Ising model and bootstrap percolation. Phys. Rev. Lett. 88, 197202 (2002)

28. Schonmann, R.H.: Critical points of 2-dimensional bootstrap percolation-like cellular automata. J. Stat. Phys. 58, 1239-1244 (1990)

29. Schonmann, R.H.: On the behavior of some cellular automata related to bootstrap percolation. Ann. Probab. 20, 174-193 (1992)

30. Treaster, M., Conner, W., Gupta, I., Nahrstedt, K.: ContagAlert: using contagion theory for adaptive, distributed alert propagation. In: Fifth IEEE International Symposium on Network Computing and Applications, pp. 126-136 (2006)

31. van Enter, A.C.D.: Proof of Straley's argument for bootstrap percolation. J. Stat. Phys. 48, 943-945 (1987) 